\title{
Zum Lernen verdammt
}

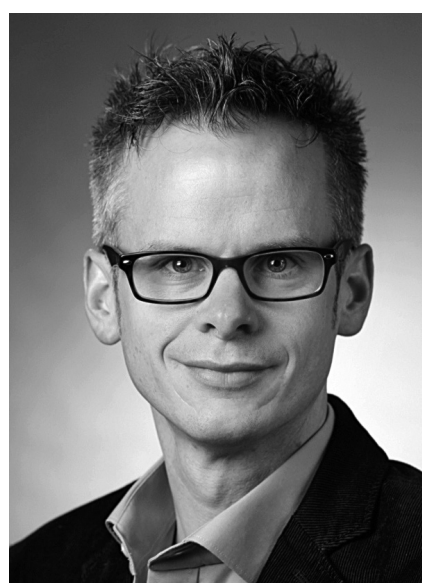

VON STEFAN GESMANN

Stefan Gesmann ist Leiter des Referats Weiterbildung an der Fachhochschule Münster, Fachbereich Sozialwesen.

s.gesmann@fh-muenster.de

\author{
Die betriebliche Weiterbildung kann als Herzstück \\ der Personalentwicklung dazu beitragen, \\ Umweltveränderungen frühzeitig wahrzunehmen \\ und das Unternehmen fit zu machen für die Zukunft. \\ Die Führungskräfte haben dabei als "Türsteher der \\ Organisation « eine besondere Verantwortung.
}

Nicht erst seit der »neoliberalen Wende« (Schubert 2005, S. 10) der 1990er Jahre stehen Leitungskräfte in Einrichtungen der Sozialen Arbeit vor einem Dilemma: Einerseits gilt es auf Grund von radikalen Veränderungen in der Organisationsumwelt (stichwortartig seien hier nur Schuldenbremse, Konkurrenzdruck, Wirkungsorientierung, demografischer Wandel und Fachkräftemangel genannt) entsprechende Strukturen, Routinen, Standardprozesse innerhalb der Organisation auszubilden, um die hohe Umweltkomplexität auf ein zu bearbeitendes Maß zu reduzieren. Andererseits führen eben jene komplexitätsreduzierenden Mechanismen dazu, dass Organisationen einen strukturellen Konservatismus ausbilden, also nur solche Informationen und Impulse an sich heran lassen, die dem bisherigen konstruierten Sinnsystem der Organisation entsprechen (vgl. Merchel 2004, S. 115).

Dies hat zur Folge, dass Organisationen in gewisser Weise träge werden und ihre Lernbereitschaft, also die Bereitschaft, die bestehenden Routinen, Handlungsmuster etc. $\mathrm{zu}$ verändern, sinkt (vgl. Wimmer 2004, S. 213). Bezeichnet man eben jene komplexitätsreduzierenden Routinen und Muster als Wissen der Organisation, dann wird nachvollziehbar, warum bisweilen pointiert festgestellt wird, dass Wissen dumm oder zumindest lernbehindert macht (vgl. Simon 2010, S. 156).

In einer Organisationsumwelt, die sich in einer bis dato nicht gekannten Geschwindigkeit verändert, kann sich keine Einrichtung der Sozialen Arbeit eine solche Lernbehinderung auf Dauer erlauben. Um innerhalb des skizzierten Dilemmas aus Stabilität und Wandel, Verändern und Bewahren, Lernen und Nicht-Lernen die richtige Balance zu finden gilt es, Formen der »maßvollen Irritation « (Merchel 2004, S. 117) in die eigenen Strukturen und Abläufe zu integrieren, um so eine dauerhafte Veränderungsbereitschaft innerhalb der Organisation aufrecht zu halten (was nicht mit dauerhaften Veränderungen gleichzusetzen ist!). Ein besonderes Irritationspotenzial kann hierbei der betrieblichen Weiterbildung attestiert werden.

\section{Die betriebliche Weiterbildung als Irritationslieferant}

Auch wenn ein Blick in die Praxis der Sozialen Arbeit dies nicht immer vermuten lässt, dient die betriebliche Weiterbildung keinem Selbstzweck, sondern zur Unterstützung der betrieblichen Zielerreichung (vgl. Berthel und Becker 2010, S. 398).

Einrichtungen der Sozialen Arbeit sind Anbieter von personenbezogenen Dienstleistungen. Die jeweilige Qualität der erbrachten Dienstleistung hängt bei solchen "front-line organizations « hochgradig von den Qualifikationen, Kompetenzen und der Leistungsbereitschaft derjenigen $a b$, die in vorderster Reihe tätig sind (vgl. Merchel et al. 2012, S. 21). Da sich, wie erwähnt, die Umwelt von Einrichtungen der Sozialen Arbeit und damit auch die Lebenswelt der Kli- 
enten von Einrichtungen der Sozialen Arbeit ständig verändert, kann es als vornehmliche Zielstellung der betrieblichen Weiterbildung betrachtet werden, die vorhandenen Qualifikationen und Kompetenzen der Mitarbeiter an sich verändernde Bedingungen anzupassen.

Neben dieser Anpassungsfunktion bieten Angebote der betrieblichen Weiterbildung dem Einzelnen jedoch zugleich die Möglichkeit »in relativer Distanz zu den Zwängen und Handlungsnotwendigkeiten des Alltags seine Wirklichkeitskonstruktion zu überdenken, mit anderen $\mathrm{zu}$ vergleichen, durch neues Wissen anzureichern, neue Sichtweisen kennenzulernen « (Arnold und Siebert 2006, S. 118). Solche »Kontingenzerfahrungen « (Wollnik 1994, S. 149) scheinen besonders in externen Angeboten der betrieblichen Weiterbildung gefördert werden zu können, wenn unterschiedliche Professionen aus unterschiedlichen funktion möglicherweise auch zur Steigerung der organisationalen Lernfähigkeit beitragen.

\section{Das leidige Transferproblem}

Bei der Analyse von Studien zum Lerntransfer kann konstatiert werden, dass gelingender Lerntransfer eher die Ausnahme als die Regel darstellt. Wenngleich unterschiedliche Studien unterschiedliche Bezugspunkte wählen, kommen sie allesamt zu dem ernüchternden Ergebnis, dass 80 bis 90 Prozent der erlernten Weiterbildungsinhalte nicht in den beruflichen Alltag transferiert werden (vgl. Kauffeld 2010, S. 4; Hummel 2001, S. 65).

Die Gründe für den mangelnden Lerntransfer sind vielschichtig und komplex. Orientierung im Dschungel von potenziellen Transferbarrieren bieten Broad und Newstrom (vgl. Broad und

\section{"Mit einer smaßvollen Irritations kann die dauerhafte Veränderungsbereitschaft in einer Organisation aufrechterhalten werden"}

Institutionen in einem geschützten Rahmen abseits des beruflichen Alltags aufeinandertreffen und fallunabhängig und ausgestattet mit zeitlichen Ressourcen ins Gespräch kommen.

Nicht selten entwickeln sich in den (Pausen-) Gesprächen mit Kollegen aus anderen Institutionen neue Sichtweisen, die zwar keinen unmittelbaren Zusammenhang zu den Weiterbildungsinhalten aufweisen, dadurch aber nicht weniger bedeutsam sind, da sie dazu einladen, nicht nur das eigene berufliche Handeln, sondern auch die diesem Handeln zu Grunde liegenden Standards, Abläufe, Routinen und Normen kritisch zu reflektieren.

Gelingt es, nicht nur das erlernte Wissen und die erlernten Methoden, sondern auch die möglicherweise irritierenden Erfahrungen abseits der klassischen Weiterbildungsinhalte in die Organisation zu transferieren und hier zum Gegenstand der Kommunikation zu machen, können Angebote der betrieblichen Weiterbildung neben ihrer primären Anpassungs-
Newstorm 1998), die in Anlehnung an Baldwin und Ford (vgl. Baldwin und Ford 1988) mit Blick auf den Lerntransfer drei zentrale Einflussfaktoren identifiziert haben:

(1) der jeweilige Weiterbildungsteilnehmer, der nicht nur über eine entsprechende Lernmotivation, sondern auch Transfermotivation verfügen sollte

(2) das Weiterbildungssetting, das bestmöglich auf den beruflichen Alltag der Teilnehmenden ausgerichtet sein sollte und

(3) der Vorgesetzte und die Kollegen (als Arbeitsumgebung zusammengefasst), die den Einzelnen bei der Anwendung des Erlernten unterstützten sollten.

Diesen drei Transferprotagonisten ordnen Broad und Newstrom drei unterschiedliche Interventionszeitpunkte zu, um Einfluss auf den Lerntransfer zu nehmen: vor Beginn einer Weiterbildung, während einer Weiterbildung und nach einer Weiterbildung. In ihren Arbeiten stellen Broad und Newstrom nicht nur unterschiedliche Ansätze vor, mit deren Hilfe innerhalb der neun Felder der Transfer-Matrix Einfluss auf den Lerntransfer genommen werden kann, sondern sie zeigen auch, dass vor allem die Arbeitsumgebung unzureichend in den Transferprozess eingebunden ist (vgl. Broad und Newstorm 1998, S. 54). Auch Köster kommt in seiner Untersuchung zu dem Ergebnis, dass die "Barrieren des Trainingstransfers (...) nicht vorwiegend in Design und Didaktik von Trainingsprogrammen zu suchen (sind, S. G.), sondern vielmehr am Arbeitsplatz des Teilnehmers selbst« (Köster 2003, S. 263).

\section{Der Weiterbildungs-Tunnelblick}

Blickt man in die Praxis der Sozialen Arbeit, so kann auch hier ein Weiterbildungstunnelblick konstatiert werden, der sich darin niederschlägt, dass innerhalb der Arbeitsumgebung den Rahmen gebenden Phasen vor Beginn und nach Beendigung einer Weiterbildung kaum Beachtung geschenkt wird und Angebote der betrieblichen Weiterbildung aus Leitungsperspektive oftmals ausschließlich auf die Phase der Durchführung reduziert werden.

Während vor Beginn der Weiterbildung nicht selten das Windhundverfahren (wer zuerst kommt, mahlt zuerst) oder das Wunschprinzip (wer hat noch nicht, wer will noch mal) gilt, wird nach der Weiterbildung der Weiterbildungstransfer weitgehend als Selbstläufer betrachtet und in die alleinige Verantwortung des sich weitergebildeten Kollegen gelegt.

Implizit wird hier ein triviales Transferverständnis zu Grunde gelegt, bei dem davon ausgegangen wird, dass der Input (die Motivation des Einzelnen die Weiterbildungsinhalte umzusetzen) linear-kausal den Output (die tatsächliche Umsetzung der Weiterbildungsinhalte) determiniert. Während sich ein solches triviales Transferverständnis bei der Übertragung von Dingen (z. B. innerhalb des Geldtransfers) durchaus bewährt, scheint es beim Weiterbildungstransfer zu versagen, da hier der sich weitergebildete Mitarbeiter auf ein soziales System trifft (die Organisation bzw. Organisationseinheit, in der er tätig ist), das sich in der Regel alles andere als trivial verhält.

Im Zuge ihres strukturellen Konservatismus schenken soziale Systeme nur 
solchen Impulsen Beachtung, die aus ihrer Sicht brauchbar erscheinen, die also an die bestehende »innere Logik" (Merchel 2005, S. 52) anschließen können. Fällt diese Brauchbarkeitsbewertung negativ aus, mobilisiert das soziale System entsprechende Abwehrmechanismen, die sich mit Blick auf die betriebliche Weiterbildung in Aussagen wie: »Das kannst du hier nicht umsetzen! oder »Das haben wir schon immer so gemacht! « niederschlagen.

Die Konsequenz ist Frustration und Ärger auf allen Seiten: Der weitergebildete Kollege ist frustriert, weil er sein neues Wissen nicht anwenden kann, die Kollegen aus dem Team sind verärgert, weil mal wieder jemand nach einer Weiterbildung meint, alles ändern zu müssen und die Leitungskraft ist frustriert und verärgert, da die Weiterbildung nicht die erhoffte Kompetenzanpassung beim Mitarbeiter bewirkt hat. Über die finanzielle Fehlinvestition und der beraubten Möglichkeit, die individuelle Lernerfahrung des Einzelnen auch zur Steigerung der organisationalen Lernfähigkeit zu nutzen, wird meist gar nicht erst nachgedacht.

\section{Leitungskräfte brauchen "Routinen zur Veränderungen von Routinen"}

Leitungskräfte sind qua Rolle nicht nur für die kurzfristige Zielerreichung, sondern vor allem für das langfristige Überleben von Organisationen innerhalb der Sozialen Arbeit zuständig. Um eben jenes organisationale Überleben in einer sich rasant verändernden Umwelt zu sichern, braucht es neben der ständigen Anpassung von Mitarbeiterkompetenzen zugleich »Routinen zur Veränderung von Routinen «(Simon 2007, S. 65). Ansätze der Personal- und Organisationsentwicklung bieten Leitungskräften Hilfestellung, um sowohl das Eine als auch das Andere zu managen.

Solange jedoch Prozesse der Organisationsentwicklung ausschließlich auf der Leitungsebene initiiert werden und die Personalentwicklung lediglich als ausführendes Organ der Organisationsentwicklung betrachtet wird, droht die Gefahr, relevante Veränderungen in der Organisationsumwelt zu spät zu erkennen oder sie gänzlich zu verschlafen.

Die betriebliche Weiterbildung als Herzstück der Personalentwicklung kann dazu beitragen, dass solche Umweltveränderungen frühzeitig wahrgenommen werden, wodurch die Personalentwicklung nicht länger nur ausführendes Organ, sondern zugleich Motor für Organisationsentwicklungsprozesse werden kann. Um dieses brach liegende Potenzial nutzen zu können, braucht es jedoch eine Abkehr vom Weiterbildungstunnelblick und dem hiermit verbundenen trivialen Transferverständnis. Solange Leitungskräfte davon ausgehen, dass sich der Transfer schon irgendwie von alleine einstellen wird, folglich keinerlei Transferunterstützung innerhalb der Arbeitsumgebung angeboten wird, um Anschlussfähigkeit zwischen Altem (»innere Logik«) und Neuem zu ermöglichen, werden sowohl die hohen Transferverluste als auch das hohe Maß an Frustration auf allen Seiten bestehen bleiben.

Wenn die weiterbildungsbedingten Lern- und Irritationserfahrungen des Einzelnen auch zur maßvollen Irritation der Handlungsroutinen, Standardprozesse etc. innerhalb der Organisation genutzt werden sollen, braucht es darüber hinaus ein erweitertes Transferverständnis, das nicht nur den Transfer an den eigenen Arbeitsplatz forciert (Transfer I. Ordnung), sondern darüber hinaus eine Überwindung der »traditionellen Individuumszentrierung " (Arnold 1995, S. 359) anstrebt und einen Lerntransfer auch in das soziale System ermöglicht (Transfer II. Ordnung). Hierbei geht es insbesondere um die Einbindung von Teams, die als zentraler Verknüpfungspunkt zwischen individuellem und organisationalem Lernen betrachtet werden können (vgl. Merchel 2005, S. 148).

Vorgesetzte haben auch hier eine Schlüsselrolle, da sie als »Türsteher der Organisation « (Köster 2003, S. 259) zumindest entsprechende Rahmenbedingungen schaffen können.

\section{Literatur}

Arnold, Rolf (1995): Theorie und Praxis des Systemischen Lernens. In: Harald Geißler (Hg.): Organisationslernen und Weiterbildung. Die strategische Antwort auf die Herausforderungen der Zukunft. Neuwied: Luchterhand, S. 352-361. Arnold, Rolf; Siebert, Horst (2006): Konstruktivistische Erwachsenenbildung. Von der Deutung zur Konstruktion von Wirklichkeit. Baltmannsweiler: Schneider-Verl. Hohengehren. Baldwin, Timothy T.; Ford, Kevin J. (1988): Transfer of Training: A Review and directions for future Research. In: Personal Psychology (41), S. 63-105.
Berthel, Jürgen; Becker, Fred G. (2010): Personal-Management. Grundzüge für Konzeptionen betrieblicher Personalarbeit. Stuttgart: Schäffer-Poeschel.

Broad, Marry L.; Newstorm, John W. (1998): Transfer of Training. Action-Packed Strategies to Ensure High Payoff from Training Investments. New York: Perseus Books.

Hummel, Thomas R. (2001): Erfolgreiches Bildungscontrolling. Praxis und Perspektiven. Heidelberg: Sauer.

Kauffeld, Simone (2010): Nachhaltige Weiterbildung. betriebliche Seminare und Trainings entwickeln, Erfolge messen, Transfer sichern. Heidelberg: Springer.

Köster, Marco (2003): Warum Training selten funktioniert. Über die Notwendigkeit von soziologischer Perspektive in einer boomenden Branche. In: Sozialwissenschaften und Berufspraxis (SuB), Bd. 26, S. 255-267.

Merchel, Joachim (2004): Qualitätsmanagement in der Sozialen Arbeit. Ein Lehr- und Arbeitsbuch. Weinheim: Juventa-Verlag. Merchel, Joachim (2005): Organisationsgestaltung in der Sozialen Arbeit. Grundlagen und Konzepte zur Reflexion, Gestaltung und Veränderung von Organisationen. Weinheim: Juventa-Verlag.

Merchel, Joachim; Pamme, Hildegard; Khalaf, Adam (2012): Personalmanagement im Allgemeinen Sozialen Dienst. Standortbestimmung und Perspektiven für Leitung. Weinheim:

Juventa-Verlag.

Schubert, Herbert (2005): Sozialmanagement zwischen Wirtschaftlichkeit und fachlichen Zielen - eine Einführung. In: Herbert Schubert (Hg.): Sozialmanagement. zwischen Wirtschaftlichkeit und fachlichen Zielen. Wiesbaden: VS Verl. für Sozialwissenschaften, S. 7-26. Simon, Fritz B. (2007): Einführung in die systemische Organisationstheorie. Heidelberg: Carl-Auer Verlag.

Simon, Fritz B. (2010): Die Kunst, nicht zu lernen. Und andere Paradoxien in Psychotherapie, Management, Politik. Heidelberg: Carl-Auer Verlag. Wimmer, Rudolf (2004): Organisation und Beratung. Systemtheoretische Perspektiven für die Praxis. Heidelberg: Carl-Auer Verlag. Wollnik, Michael (1994): Interventionschancen bei autopoietischen Systemen. In: Klaus Götz (Hg.): Theoretische Zumutungen. Vom Nutzen der systemischen Theorie für die Managementpraxis. Heidelberg: Carl-Auer Verlag S. 118-160. 